\title{
Transition from single-domain to vortex state in soft magnetic cylindrical nanodots
}

\author{
W. Scholz,${ }^{1, *}$ K. Yu. Guslienko, ${ }^{2}{ }^{\dagger}$ V. Novosad,${ }^{3}$ D. Suess, ${ }^{1}$ T. Schrefl, ${ }^{1}$ R. W. Chantrell, ${ }^{2}$ and J. Fidler ${ }^{1}$ \\ ${ }^{1}$ Vienna University of Technology, Wiedner Hauptstrasse 8-10/138, A-1040 Vienna, Austria \\ ${ }^{2}$ Seagate Research, 1251 Waterfront Place, PA 15222-4215 \\ ${ }^{3}$ Material Science Division, Argonne National Laboratory, 9700 S. Cass Ave., Argonne, IL 60439
}

(Dated: September 24, 2002)

\begin{abstract}
We have investigated the magnetic properties of submicron soft magnetic cylindrical nanodots using an analytical model as well as three dimensional numerical finite element simulations. A detailed comparison of the magnetic vortex state shows the differences between these two models. It appears that the magnetic surface charges play a crucial role in the equilibrium magnetization distribution especially for shifted vortices. In addition magnetic volume charges, which arise from a radial component of the magnetization, have been found. Finally, the magnetic phase diagram for soft magnetic particles with varying aspect ratio is presented.
\end{abstract}

\section{INTRODUCTION}

The recent advances in microfabrication techniques have stimulated interest in the properties of submicron sized patterned magnetic elements. ${ }^{1,2}$ Promising applications include magnetic random access memory, high-density magnetic recording media, and magnetic sensors. ${ }^{3}$ However, in order to exploit the special behavior of magnetic nanoelements it is necessary to study and understand their fundamental properties. We have studied the static properties of cylindrical magnetic nanodots of different sizes and aspect ratios with analytical models and numerical finite element (FE) simulations, especially magnetic vortex states.

Direct experimental evidence for the existence of these magnetic vortex states has been found by the method of magnetic force microscopy. Shinjo and coworkers ${ }^{4}$ studied magnetic nanodots of permalloy $\left(\mathrm{Ni}_{80} \mathrm{Fe}_{20}\right)$ with a thickness of $50 \mathrm{~nm}$ and a radius between $300 \mathrm{~nm}$ and $1 \mu \mathrm{m}$, for example. Their hysteresis loops have be measured by vibrating sample magnetometer ${ }^{2}$ and magnetooptical methods ${ }^{5}$ and successfully identified single domain and vortex states. Furthermore, these magnetic vortex states are an interesting object for high frequency magnetization dynamics ${ }^{6}$ experiments, which are important for high-density magnetic recording media, where high-frequency field pulses of the magnetic write head store the information by reversing the magnetization.

\section{THE ANALYTICAL VORTEX MODEL}

The rigid vortex model assumes a "rigid vortex", which does not change its shape in an external field. Together with a certain magnetization distribution it gives an approximation for the magnetization distribution of a curling state (vortex state) in a fine cylindrical particle. An analytical model for the magnetization distribution $\mathbf{M}(\mathbf{x})$ in zero field has been developed using a variational principle by Usov and coworkers. ${ }^{7,8}$ It is split into two parts. The first part describes the magnetization in the core of the vortex $(r \leq a)$, which is defined by $M_{z} \neq 0$ (if the dot lies in the $x / y$-plane with its cylinder axis parallel to the $z$-axis).

$$
\begin{aligned}
& M_{x}=-\frac{2 a r}{a^{2}+r^{2}} \sin \varphi \\
& M_{y}=\frac{2 a r}{a^{2}+r^{2}} \cos \varphi \\
& M_{z}=\sqrt{1-\frac{2 a r}{a^{2}+r^{2}}}
\end{aligned}
$$

The other describes the magnetization outside the core $(r>a)$.

$$
\begin{aligned}
& M_{x}=-\sin \varphi \\
& M_{y}=-\cos \varphi \\
& M_{z}=0
\end{aligned}
$$

The transition is continuous but not differentiable and defines the vortex core radius $c$. Eqs. 1 and 2 represent a two-dimensional radially symmetric vortex model, which has the following properties: 1) In equilibrium in zero field there are surface charges only on the top and bottom surface within the vortex core. 2) For shifted vortices surface charges are induced on the circumference of the nanodot. 3) There are no volume charges.

In this work we have checked the applicability of Usov's analytical vortex model and the rigid vortex model in an external field numerically. In addition we have studied the dependence of $M(r)$ on the $z$-coordinate (along the thickness of the nanodot).

\section{THE FINITE ELEMENT MODEL}

The numerical computer simulations have been carried out using a 3D dynamic hybrid finite element/boundary element micromagnetic code with a static scalar potential for the calculation of the demagnetizing field and a preconditioned backward differentiation formula for the time integration of the Landau-Lifshitz equation of motion. ${ }^{9}$

In our calculations we have assumed the following material parameters for permalloy $\left(\mathrm{Ni}_{80} \mathrm{Fe}_{20}\right): M_{\mathrm{s}}=$ $8 \times 10^{5} \mathrm{~A} / \mathrm{m}=8 \times 10^{2} \mathrm{G}, A=13 \times 10^{-12} \mathrm{~J} / \mathrm{m}=$ $1.3 \times 10^{-6} \mathrm{erg} / \mathrm{cm}$, anisotropy has been neglected. Thus, the exchange length is $l_{\text {ex }}=\sqrt{2 A /\left(\mu_{0} M_{\mathrm{s}}^{2}\right)}=5.7 \mathrm{~nm}$. 


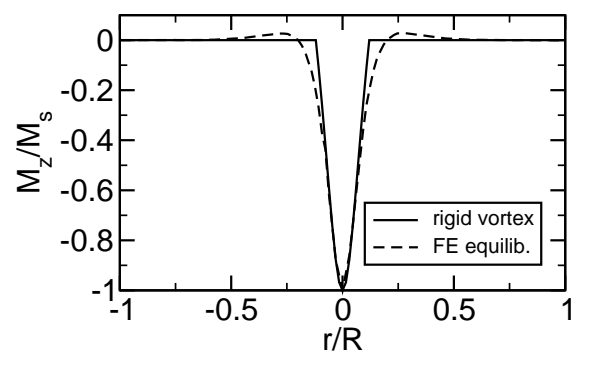

FIG. 1: Comparison of the profiles of $M_{z}$ between the analytical model and the remanent state obtained by the finite element simulation. The profile has been taken along the $x$ axis through the center of the nanodot, which has an aspect ratio of $L / R=20 \mathrm{~nm} / 100 \mathrm{~nm}$.

Fig. 1 shows the $M_{z}$ profile of a nanodot with a radius of $100 \mathrm{~nm}$ and a thickness of $20 \mathrm{~nm}$ as obtained by the analytical vortex and the finite element model in zero field in equilibrium.

The results show, that the vortex core is approximately $54 \%$ larger $(18.5 \mathrm{~nm})$ than assumed by the analytical vortex model $(12 \mathrm{~nm})$, if the core radius is defined by the first $M_{z}=0$ crossover from the center. Furthermore there is a region with $M_{z}<0$ outside the vortex core. Thus, we find positive surface charges in the core of the vortex, which are surrounded by negative surface charges. Only outside of approximately half the radius $(50 \mathrm{~nm})$ almost all surface charges disappear. It has been verified, that there is very little variation of the magnetization distribution across the thickness of the nanodot.

Similar magnetization profiles have been calculated by Buda et al. ${ }^{10}$ for circular Co dots with perpendicular magnetocrystalline anisotropy. There the uniaxial anisotropy leads to a stripe domain structure (circles around the vortex core), which gets more pronounced as the dot thickness increases.

\section{ENERGY AND HYSTERESIS}

Then the exchange and magnetostatic energies of the vortices have been compared. The formulas for the analytical vortex model have been derived by Usov et al. ${ }^{7,8}$ The finite element results are in good agreement with the analytical results and it is shown in table I, that the energy of the equilibrium magnetization distribution, which has been found with the FE model, is more than $4 \%$ smaller than that of the analytical vortex model.

Fig. 2 shows the hysteresis curve for a circular nanomagnet with in-plane external field. For very high external fields (applied in the plane of the nanodot), the magnetization is almost uniform and parallel to the external field (Fig. 3(a)). As the field decreases (solid line in Fig. 2) the magnetization distribution becomes more and more non uniform, which is caused by the magnetostatic stray field. Upon further decrease of the external field, the symmetry of the magnetization distribution breaks

\begin{tabular}{|c|c|c|c|}
\hline units: $\mathrm{J} / \mathrm{m}^{3}$ & $\begin{array}{l}\text { Magnetostatic } \\
\text { energy }\end{array}$ & $\begin{array}{l}\text { Exchange } \\
\text { energy }\end{array}$ & $\begin{array}{l}\text { Total } \\
\text { energy }\end{array}$ \\
\hline Analytical vortex model & 432.1 & 5356 & 5788 \\
\hline FE simulation (equilib.) & 387.1 & 5150 & 5537 \\
\hline difference FE - analytical & $-10.42 \%$ & $-3.85 \%$ & $-4.35 \%$ \\
\hline
\end{tabular}

TABLE I: Comparison of the energies obtained by the analytical vortex model and the numerical FE simulation.

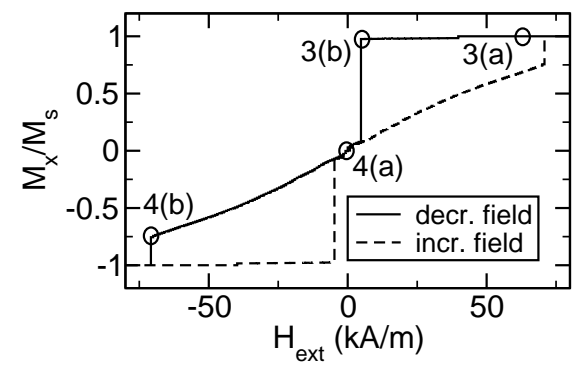

FIG. 2: Hysteresis curve of a nanodot with $L / R=$ $20 \mathrm{~nm} / 100 \mathrm{~nm}$ for an external field applied in plane. The circles mark the position on the hysteresis curve at which the snapshots in Figs. 3 and 4 have been taken.

and a "C" state (Fig. 3(b)) develops. At the nucleation field (about $5 \mathrm{kA} / \mathrm{m}$ for our example) a vortex nucleates on the circumference and quickly moves towards its equilibrium position (close to the center of the nanodot). As a result we find a sudden drop in the average magnetization. When the external field is reduced to zero the vortex moves into the center of the nanodot (Fig. 4(a)). If the external field is increased in the opposite direction, the vortex is forced out of the center of the dot. For about $-70 \mathrm{kA} / \mathrm{m}$ the vortex is pushed out of the nanodot (annihilation: Fig. 4(b)) and we find the second jump in the hysteresis curve to (almost) saturation.

This characteristic behavior has also been found experimentally using Hall-micromagnetometry by Hengstmann et al. ${ }^{11}$, who measured the stray field of individual permalloy disks using a sub- $\mu \mathrm{m}$ Hall magnetometer. The hysteresis loops of arrays of Supermalloy nanomagnets have been measured by Cowburn et al. ${ }^{5}$ using the Kerr effect. Their characteristic loop shape has then been

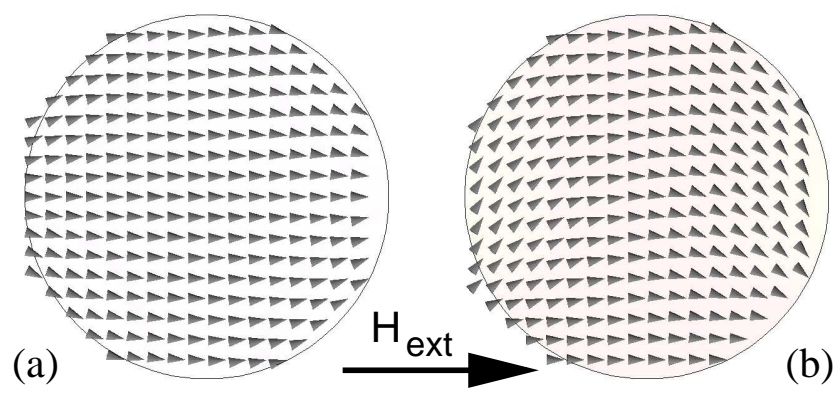

FIG. 3: Leaf state (a) (for high external in-plane fields) and "C" state (b) (develops before the vortex nucleation). 


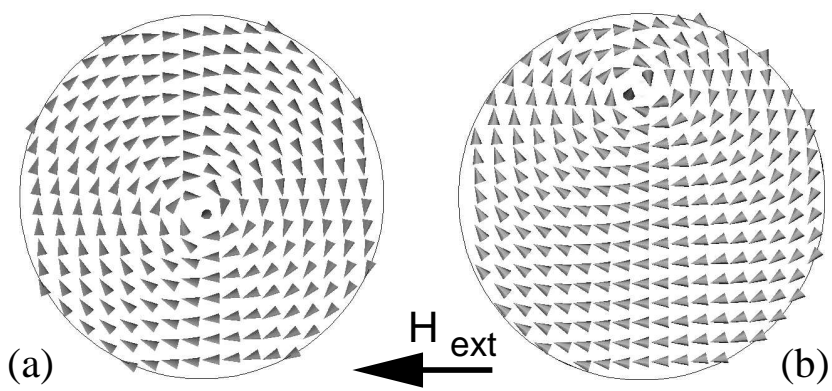

FIG. 4: Centered vortex (a) in zero field and far shifted vortex (b) before annihilation.

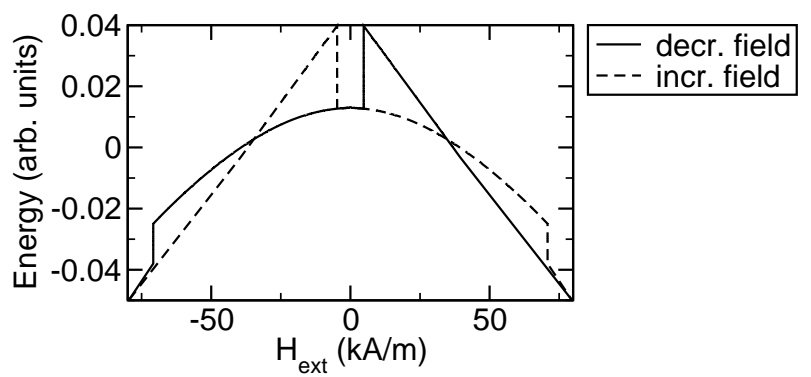

FIG. 5: Total energy as a function of the external field for both branches (solid line for decreasing field - dashed line for increasing field) of the hysteresis loop.

used to identify the single-domain in-plane and the vortex phase.

The rigid vortex model can very well describe the susceptibility and magnetization distribution for low fields as well as the vortex annihilation field. However, it cannot predict the vortex nucleation field. ${ }^{12-15}$

In Fig. 5 the total energy is plotted as a function of the external field for the branch of decreasing field of the hysteresis curve. The solid line indicates the hysteresis branch for decreasing external field and the dashed line that for increasing field. For very high fields we have an almost uniformly magnetized nanodot. As the external field decreases the total energy increases (almost) linearly. The dashed line for positive field values indicates the total energy for the vortex state. At the intersection of the solid and the dashed line (at a value of about $35 \mathrm{kA} / \mathrm{m}$ for the external field) the vortex state and the uniform magnetization have equal energy. However, they are separated by an energy barrier, which arises from the magnetostatic energy, which in turn is caused by the stray field on the circumference of the nanodot as the vortex is pushed out of the center. Thus, the vortex state is a metastable state for external fields higher than $35 \mathrm{kA} / \mathrm{m}$ and the uniform state is metastable for external fields below $35 \mathrm{kA} / \mathrm{m}$.

The field dependence of exchange and magnetostatic energy are given in Fig. 6. The exchange energy remains approximately constant for negative external fields until the annihilation field is reached. Since all exchange energy is stored in the vortex core, this indicates that

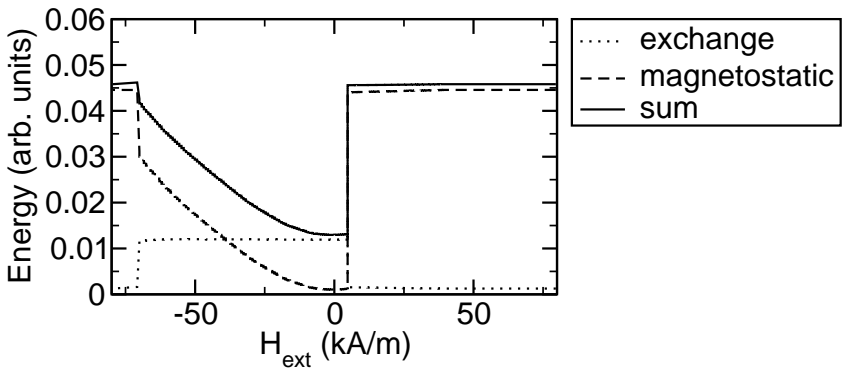

FIG. 6: Exchange and magnetostatic energy and their sum as a function of the external field (for decreasing external field).

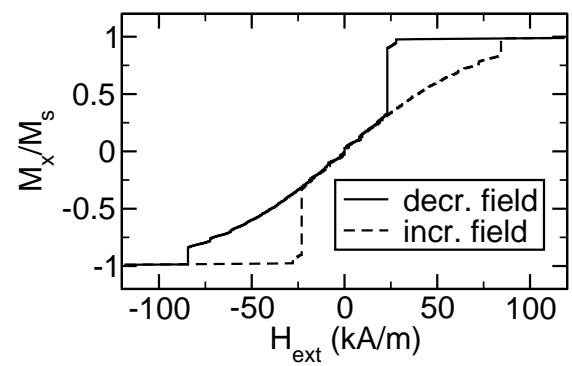

FIG. 7: Hysteresis curve of a nanodot with $L / R=$ $40 \mathrm{~nm} / 200 \mathrm{~nm}$.

the vortex core remains undisturbed for even very large vortex shifts.

For a twice as large nanodot with $R=200 \mathrm{~nm}$ and $L=40 \mathrm{~nm}$ we find a nucleation field of $28 \mathrm{kA} / \mathrm{m}$ and an annihilation field of $84 \mathrm{kA} / \mathrm{m}$. The corresponding hysteresis loop is given in Fig. 7 .

\section{AVERAGE MAGNETIZATION}

Fig. 8 shows profiles of $M_{z}$ along the $y$-axis for different external fields. As a result, the vortex is shifted and the profile "moves" towards the circumference of the dot $(|y / R|=1)$. From this plot the position of the vortex core for a given external field has been extracted. The corresponding average magnetization $\left\langle M_{x}\right\rangle$ is plotted in Fig. 9 (open circles). For symmetry reasons $M_{y}$ is zero (the vortex is shifted along the $y$-axis, since we applied a field in $x$-direction).

By integrating the magnetization distribution of the rigid vortex model over the surface of the nanodot the average magnetization $\left\langle M_{x}\right\rangle$ has been calculated. The result is given in Fig. 9. We find very good agreement between the rigid vortex model and the finite element simulation and the small difference can be understood by considering small deviations of the magnetization distribution due to surface charges on the circumference (cf. section VI). 


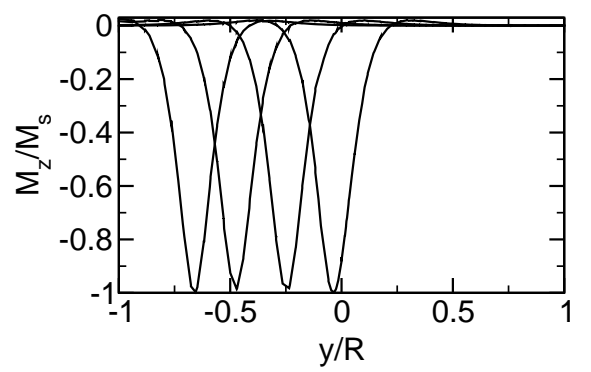

FIG. 8: Profiles of $M_{z}$ along the $y$-axis through the center of the nanodot for a vortex moving in $-y$ direction due to an external field increasing in $x$ direction.

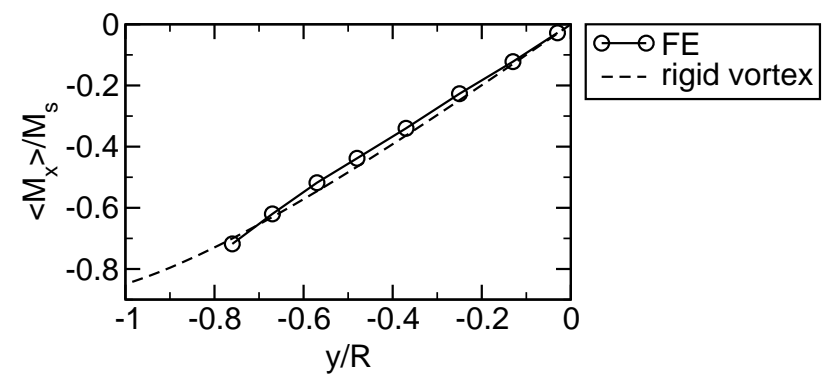

FIG. 9: Comparison of $\left\langle M_{x}\right\rangle$ as a function of the vortex displacement between the $\mathrm{FE}$ simulation and the rigid vortex model. $y=0$ corresponds to a centered vortex, $y=-0.78$ is the maximum shift before vortex annihilation occurs in the FE simulation.

\section{CHARGE DENSITIES AND THE MAGNETOSTATIC FIELD}

Another important aspect for comparison with the rigid vortex model is the magnetostatic energy, and the surface and volume charges, which generate the magnetostatic field. On the top and bottom circular surface the surface charges are proportional to $M_{z}$, because the normal vector $\mathbf{n}$ of the top and bottom is simply $e_{z}$ and $-e_{z}$, respectively. However, on the circumference the normal vector is, of course, $e_{r}$.

If an in-plane external field is applied, the vortex core is shifted perpendicular to the direction of the field (Fig. 4). As a result, surface charges appear on the circumference.

The surface charge densities for different vortex core displacements, as calculated with the FE model, are given in Fig. 10. For small external fields and therefore small vortex displacements there is very good agreement between the rigid vortex model and the finite element simulation. As the external field increases more surface charges appear on the circumference of the nanodot. However, the rigid vortex model overestimates these surface charges. The values for the average magnetization is in good agreement, but the surface charge distribution is not. The reason is, that the magnetization distribution close to the circumference is disturbed by the strong demagnetizing fields. As we further increase the external

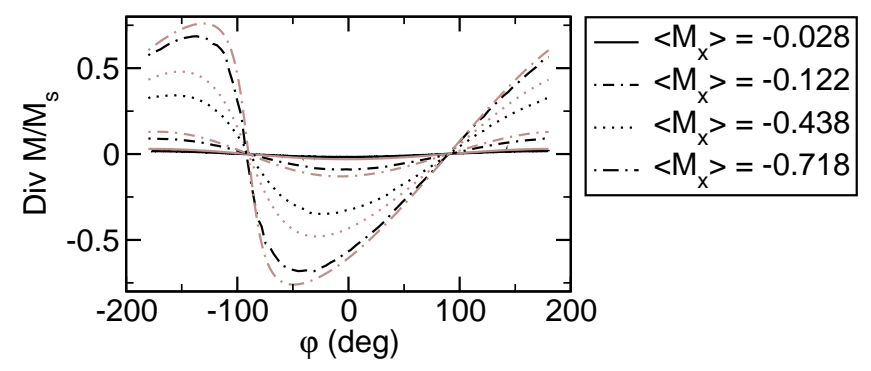

FIG. 10: Surface charge distribution on the circumference of the nanodot as a function of the polar angle. The rigid vortex model (gray lines) overestimates the charge density as compared to the FE simulation (black lines).

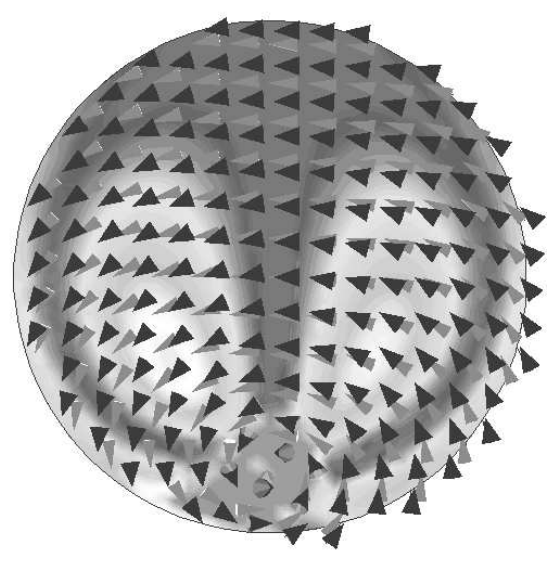

FIG. 11: Contour plot of $d=\left|\mathbf{M}_{\mathrm{FE}}-\mathbf{M}_{\mathrm{rv}}\right|$. Dark areas indicate good agreement of the magnetization distribution between the rigid vortex model (black cones) and the FE model (gray cones), lighter areas indicate larger differences.

field and the vortex displacement this deviation becomes more and more pronounced and in addition to that we also find some deviation in the center of the nanodot, which arises from a more "elliptical" shape of the magnetization distribution as the vortex is pushed towards the boundary. A contour plot of the difference between the the magnetization distribution calculated by the $\mathrm{FE}$ simulation and the rigid vortex model $d=\left|\mathbf{M}_{\mathrm{FE}}-\mathbf{M}_{\mathrm{rv}}\right|$ is shown in Fig. 11 for $H_{\text {ext }}=66.0 \mathrm{kA} / \mathrm{m}=830 \mathrm{Oe}$, $\left\langle M_{x}\right\rangle / M_{\mathrm{s}}=-0.72$, and $b / R=-0.76$. The light areas at the circumference and in the center of the nanodot indicate differences between the rigid vortex model and the FE simulation.

The $3 \mathrm{D}$ isosurface image of $\operatorname{div} \mathrm{M} / M_{s}$ in Fig. 12 shows the density of volume charges in the nanodot. The maximum and minimum values of $\operatorname{divM} / M_{s}$ were about $-2.5<\operatorname{div} \mathrm{M} / M_{s}<+2.5$. Similar to the results for the surface charge density, which has been discussed above, we find positive volume charges just below the vortex core and in a "ring" around the core on the opposite side. The negative volume charges appear below the vortex on the bottom and in a ring around the positive vortex core on top (Fig. 12). 


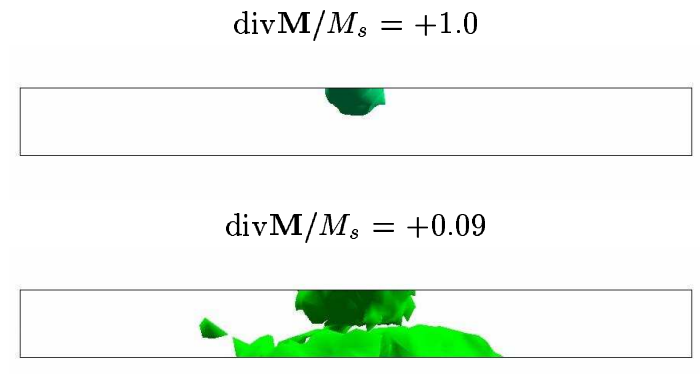

FIG. 12: 3D isosurface plots of the volume charge density in permalloy nanodots (side view).

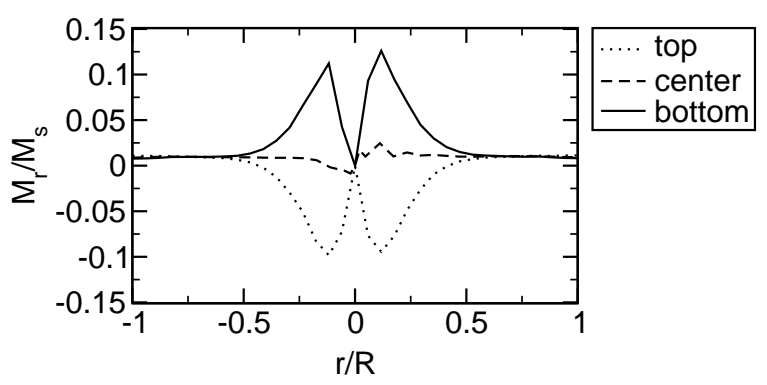

FIG. 13: $M_{r}$ line scan across the nanodot at its top, in the center, and at the bottom.

Since $M_{z}$ is very uniform along the thickness of the nanodot, the radial component of the magnetization $M_{r}$ is responsible for the volume charges (Fig. 13).

The demagnetizing field arising from the vortex structure is mainly concentrated in the vortex core (Fig. 14). It has a dominating $z$ component and a smaller radial component.

\section{PHASE DIAGRAM}

A summary of the results of the equilibrium magnetization distribution of nanodots with different aspect ratios is given in the phase diagram in Fig. 15. The transition from the in-plane magnetization to the vortex state is very sharp, because this requires that the symmetry of the single domain state with (almost) homogeneous

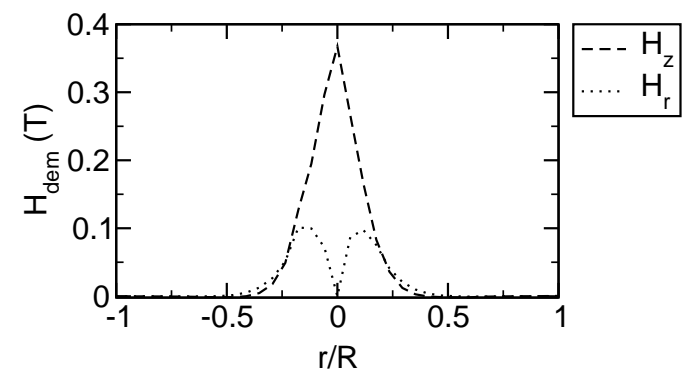

FIG. 14: $H_{z}^{\text {dem }}$ and $H_{r}^{\text {dem }}$ across the nanodot.

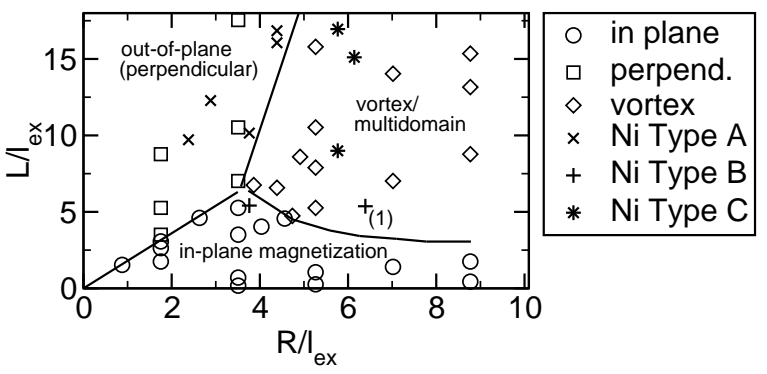

FIG. 15: Phase diagram of magnetic ground states (axis scaling in units of the exchange length). The data points indicated by the open symbols have been calculated. The experimental data have been taken from Ross et al. "Ni Type A" indicates Ni samples with out-of-plane (perpendicular) magnetization at remanence, "Ni Type B" indicates in-plane, and "Ni Type C" vortex or multidomain states, respectively. The experimental data nicely fit in the phase diagram with one exception, which is indicated by "(1)". There, a remanent state with in-plane magnetization has been found, where a vortex state might be expected. The solid lines just separate the different regions from each other.

in-plane magnetization breaks in order to form the vortex state with cylindrical symmetry. The slope of the line separating the in-plane and out-of-plane remanent states has a slope of 1.80 , which is in agreement with the simulations by Ross et al. ${ }^{2}$ and analytical calculations. ${ }^{16}$ Magneto-optical measurements of hysteresis curves ${ }^{5}$ also show a distinct change between these two regimes: The single domain particles retain high remanence and switch at very low fields, whereas a sudden loss in magnetization (cf. Fig. 2) as the external field is reduced is typical of a flux closure configuration (vortex state). However, the transition from the vortex to the perpendicular magnetization (parallel to the cylinder axis) is not well defined. The numerical experiments show a smooth transition from one state to the other. For decreasing aspect ratio, the magnetization starts to twist and exhibits very inhomogeneous magnetization distributions. The two-dimensional analytical model cannot describe this transition properly, because it would require, that the dependence of the magnetization on the $z$-coordinate is taken into account. In the phase diagram (Fig. 15) a magnetization distribution with $M_{z}>0.75$ has been defined as being a perpendicular ground state.

Experimental data this have been obtained from arrays of soft magnetic cylindrical particles by Ross et al. ${ }^{2}$ The data of their Ni samples are also shown in Fig 15. The agreement with the numerically calculated phase diagram is very good. Only one data point does not fit in: A remanent state with in-plane magnetization is found, where a vortex state might be expected. However, also the smooth transition from the perpendicular magnetization to the vortex (multidomain) state has been found. 


\section{CONCLUSIONS}

A detailed comparison of the rigid vortex model for magnetic vortex states in soft magnetic nanodots with finite element simulations has revealed some special features of the magnetic vortex state. The magnetization distribution near the vortex core radius $(r=a)$ deviates essentially from Usov's analytical model. Especially a non vanishing radial component $M_{\rho}$ has been found (Fig. 13). In addition to the magnetic surface charges in the core of the vortex the finite element simulations have revealed a ring of weak surface charges with opposite sign around the core as well as volume charges within the nanodot. The shape of the vortex core and its exchange energy have been found to be very stable ("rigid") even for large vortex shifts in an external field. However, the surface charges on the circumference of the nanodot are overestimated by the rigid vortex model, because the magnetization distribution is distorted from its perfectly circular shape by the magnetostatic stray field. As a result, the surface charges and the magnetostatic energy are reduced as compared to the rigid vortex model. Finally, the phase diagram of magnetic ground states shows sharp transitions from the "in-plane" state to the perpendicular magnetization distribution and the magnetic vortex state, whereas the transition from the perpendicular magnetization to the magnetic vortex state is not well defined.

\section{Acknowledgments}

Work supported by the Austrian Science Fund, Y-132 PHY. The authors would like to thank M. Grimsditch for helpful discussions. R. W. Chantrell would like to acknowledge Robert Shull for handling the editorial process including the anonymous review procedure.
* werner.scholz@tuwien.ac.at; http://magnet.atp. tuwien.ac.at/scholz/

$\dagger$ Konstantin.Guslienko@seagate.com

1 C. A. Ross, Annu. Rev. Mater. Res. 31, 203 (2000).

2 C. A. Ross, M. Hwang, M. Shima, J. Y. Cheng, M. Farhoud, T. A. Savas, H. I. Smith, W. Schwarzacher, F. M. Ross, M. Redjdal, et al., Phys. Rev. B 65, 1444171 (2002).

3 R. P. Cowburn, J. Phys. D: Appl. Phys. 33, R1 (2000).

4 T. Shinjo, T. Okuno, R. Hassdorf, K. Shigeto, and T. Ono, Science 289, 930 (2000).

${ }^{5}$ R. P. Cowburn, D. K. Koltsov, A. O. Adeyeye, M. E. Welland, and D. M. Tricker, Phys. Rev. Lett. 83, 1042 (1999).

6 V. Novosad, M. Grimsditch, K. Y. Guslienko, P. Vavassori, Y. Otani, and S. D. Bader, Phys. Rev. B 66, 052407 (2002).

7 N. A. Usov and S. E. Peschany, J. Magn. Magn. Mater. 118, L290 (1993).

${ }^{8}$ N. A. Usov and S. E. Peschany, Fiz. Met. Metalloved (transl.: The Physics of Metals and Metallography) 12, 13 (1994).

9 D. Suess, V. Tsiantos, T. Schrefl, J. Fidler, W. Scholz, H. Forster, R. Dittrich, and J. J. Miles, J. Magn. Magn. Mater. 248, 298 (2002).

10 L. D. Buda, I. L. Prejbeanu, M. Demand, U. Ebels, and K. Ounadjela, IEEE Trans. Magn. 37, 2061 (2001).

11 T. M. Hengstmann, D. Grundler, C. Heyn, and D. Heitmann, J. Appl. Phys. 90, 6542 (2001).

12 K. Y. Guslienko, V. Novosad, Y. Otani, H. Shima, and K. Fukamichi, Appl. Phys. Lett. 78, 3848 (2001).

13 K. Y. Guslienko, V. Novosad, Y. Otani, H. Shima, and K. Fukamichi, Phys. Rev. B 65, 024414 (2001).

14 M. Schneider, H. Hoffmann, and J. Zweck, Appl. Phys. Lett. 77, 2909 (2000).

15 A. Fernandez and C. J. Cerjan, J. Appl. Phys. 87, 1395 (2000).

16 A. Aharoni, J. Appl. Phys. 68, 2892 (1990). 